\title{
DETERMINACIÓN DE LA RESISTENCIA AL CLETODIM EN Poa annua EN COSTA RICA ${ }^{1}$
}

\author{
Ana María Rodríguez-Ruiz $z^{2}$, Néstor Chaves-Barrantes ${ }^{3}$, Alberto Hernández-Díaz ${ }^{4}$, Franklin Herrera-Murillo
}

\begin{abstract}
RESUMEN
Determinación de la resistencia al cletodim en Poa annua en Costa Rica. El objetivo de este trabajo fue verificar la presencia de resistencia al cletodim en poblaciones de $P O a$ апnua, en el cultivo de cebolla en la zona alta de Cartago, Costa Rica. Se realizaron dos experimentos; el primero se llevó a cabo en el año 2007, en una casa de mallas de la Estación Experimental Agrícola Fabio Baudrit Moreno (EEAFBM), con semilla de plantas que procedieron de una finca donde cletodim ejerció buen control (S), y de otra finca con control deficiente (R). En cada población se aplicó cletodim a diferentes dosis $(0,5 \mathrm{X}, 1,0 \mathrm{X}, 1,5 \mathrm{X}$ y $2,0 \mathrm{X})$ siendo $\mathrm{X}$ la dosis comercial recomendada. En este experimento las dosis de 1,5 y 2,0X de cletodim mostraron un 44 y $17 \%$ de control respectivamente, en la población $\mathrm{R}$, mientras que las mismas dosis controlaron en $100 \%$ la población S. El segundo experimento se realizó en el 2009, recolectándose directamente en el campo semillas de dos poblaciones de $P$. annua, una en un sitio con control satisfactorio (S) y la otra de un lote de la misma finca donde el control fue deficiente (R). Las semillas se sembraron en recipientes plásticos según poblaciones y se aplicó cletodim a las dosis de $0,1 \mathrm{X}$, 2X, 4X, 8X, 16X. Se encontraron diferencias significativas entre las poblaciones de $P$. аnnua en su reacción al cletodim. En la población $\mathrm{S}$ se obtuvo mayor porcentaje de control y menos plantas sobrevivientes que la población R. Varias plantas de la población $\mathrm{R}$ soportaron hasta dieciséis veces la dosis comercial de cletodim. Los resultados de ambos experimentos sugieren la presencia de poblaciones de $P$. аппиа con resistencia a cletodim.
\end{abstract}

Palabras claves: poáceas resistentes a herbicidas, ciclohexanodionas, arvenses.

\begin{abstract}
Assessment of Poa annua resistance to clethodim in Costa Rica. The objective of this study was to check for the presence of resistance to clethodim in Pоа апnиa populations observed in onion fields in the highlands of Cartago, Costa Rica. Two experiments were conducted; the first one was carried out, in 2007, in a greenhouse at the Fabio Baudrit Moreno Agricultural Experiment Station (EEAFBM, in Spanish), with seeds of plants from a farm where clethodim exercised good control (S population), and from another farm where clethodim showed a deficient control ( $\mathrm{R}$ population). Both $\mathrm{S}$ and $\mathrm{R}$ populations were treated with different doses of clethodim $(0.5 \mathrm{X}, 1.0 \mathrm{X}, 1.5 \mathrm{X}$ and $2.0 \mathrm{X})$, being $\mathrm{X}$ the recommended commercial dose). In this experiment, control of the R population was $44 \%$ and $17 \%$ at doses of $1.5 \mathrm{X}$ and $2.0 \mathrm{X}$, respectively, whereas control of the $\mathrm{S}$ population was $100 \%$ at either of those doses. The second experiment was carried out in 2009, seeds of two other $P$. annua populations were collected in a similar way: one population from an area where weed control was satisfactory $(\mathrm{S})$, and the other population from an area of the farm where control was deficient (R). Seeds were sown, depending on the type, in plastic pots and sprayed with clethodim at doses of: $0.1 \mathrm{X}$, $2 \mathrm{X}, 4 \mathrm{X}, 8 \mathrm{X}$, and $16 \mathrm{X}$. There were significant differences in the reactions to clethodim of both $P$. аnnua populations The $S$ population showed a higher percentage of control and less surviving plants than the R population. Several plants of the R population resisted up to $16 \mathrm{X}$ commercial doses of clethodim. Results of both experiments suggest the presence of $P$. аnnua populations resistant to clethodim.
\end{abstract}

Keywords: herbicide resistant grasses, cyclohexanedione, weeds.

\footnotetext{
Recibido: 30 de enero, 2014. Aceptado: 25 febrero, 2015. Parte del proyecto de investigación 736-A8-198 de la Estación Experimental Agrícola Fabio Baudrit Moreno, inscrito en la Vicerrectoría de Investigación de la Universidad de Costa Rica.

2 Universidad de Costa Rica, Estación Experimental Fabio Baudrit Moreno, Programa de Manejo Integrado de Malezas. Alajuela, Costa Rica. ana.rodriguezruiz@ucr.ac.cr, franklin.herrera@ucr.ac.cr

3 Universidad de Costa Rica, Estación Experimental Fabio Baudrit Moreno, Programa de Leguminosas. Alajuela, Costa Rica. nestor.chaves@ ucr.ac.cr

4 Ministerio de Agricultura y Ganadería (MAG), Agencia de Extensión Agrícola. Potrero Cerrado, Cartago, Costa Rica. aherdz@ costarricense.cr
} 


\section{INTRODUCCIÓN}

Poa апnиa es una planta anual y cespitosa introducida en Costa Rica y que pertenece a la familia Poaceae (Pohl, 1980). Es considerada una maleza problemática en países como Alemania, Inglaterra, Estados Unidos y Bélgica, entre otros; se le considera como una especie con alta variación genotípica y fenotípica (Holm et al., 1977; Eelen et al., 1999; Binkholder et al., 2011). P. аппиа puede llegar a producir de 30000 a 210000 semillas por metro cuadrado en invierno o primavera respectivamente y hasta 270000 macollas en la misma área, aunque no todas lleguen a florecer (Lush, 1988a; 1988b). En Costa Rica $P$. апnиa es una maleza común en la zona alta (hortícola) de la provincia de Cartago, principalmente en los cultivos de cebolla y zanahoria.

El término resistencia, en referencia a las malezas, se define como la capacidad heredable de una planta o un biotipo de la misma de sobrevivir y reproducirse después de ser expuesta a una aplicación de herbicida en una dosis a la que normalmente la población original o silvestre era sensible (WSSA, 1998; Taberner et al., 2007). La resistencia puede aparecer en forma natural o llegar a inducirse a través de diferentes técnicas (WSSA, 1998).

Es importante distinguir entre malezas sensibles e insensibles o tolerantes; en el primer caso puede decir que no sobreviven a la aplicación de la dosis recomendada de uso de un herbicida; en tanto que en el segundo, se refiere a un determinado biotipo de maleza que nunca se ha podido controlar con un determinado herbicida (Taberner et al., 2007). La Sociedad Americana de Ciencias de Malezas (WSSA, por sus siglas en inglés) establece que la tolerancia es "la capacidad hereditaria de una especie de sobrevivir y reproducirse después del tratamiento con determinado herbicida" y que además no ha sido manipulada o sometida a selección genética para que la planta sea tolerante (WSSA, 1998).

Es relevante considerar las definiciones de resistencia cruzada y múltiple; la primera de ellas se presenta cuando un mecanismo de resistencia individual del biotipo resistente le confiere resistencia a varios herbicidas con el mismo mecanismo de acción, a la vez esta resistencia cruzada puede presentarse en el sitio de acople común a varios herbicidas o fuera de este sitio activo. La resistencia múltiple se presenta cuando en una planta o población de estas se observan dos o más mecanismos de resistencia (Valverde y Heap, 2010).

La resistencia en malezas es un fenómeno que se ha incrementado en los últimos años, desde la aparición de las primeras especies resistentes a herbicidas auxínicos (Owen, 2001) y simazina (Ryan, 1970). Para 1980 ya se conocían veintiséis especies de hoja ancha y seis de poáceas resistentes a las triazinas, en el 2009 se encontraban registrados 332 biotipos resistentes a diferentes grupos de herbicidas (Valverde y Heap, 2010) y para el 2014 se sabe de la existencia de 432 casos únicos (especies por sitio de acción) de malezas resistentes a los herbicidas a nivel mundial, con 235 especies (138 dicotiledóneas y 97 monocotiledóneas) (Heap, 2014).

Uno de los grupos de herbicidas con más casos de resistencia son los inhibidores de la Acetil-coenzima A carboxylasa (ACCasa), primera enzima en el proceso metabólico para la síntesis de lípidos (Preston y Mallory-Smith, 2001), y cuyo mecanismo de acción es el que posee el herbicida cletodim. Dentro del grupo de herbicidas que afectan la ACCasa se encuentran las siguientes familias químicas: los ariloxifenoxipropanoatos ("fops"), las ciclohexanodionas ("dim") y las fenilpirasolinas (“dens"), que afectan especialmente especies poáceas, de las cuales se encuentran reportados 36 biotipos resistentes a estos grupos de herbicidas (Preston y Mallory-Smith, 2001; Valverde y Heap, 2010).

Volenberg y Stoltenberg (2002) encontraron accesiones de Setaria faberi y Digitaria sanguinalis con resistencia cruzada de menor a mayor grado respectivamente, a los herbicidas cletodim, fluazifop-p (ácido) y setoxidim. Un caso similar es la detección de resistencia a diclofop metil, clodinafop-propargil, haloxyfop metil (grupo de los "fops") y a tralkoxidim, cletodim y setoxidim (grupo de los "dim") en un biotipo de Lolium rigidum el cual ya era resistente a haloxyfop metil, donde el grado de resistencia fue mayor en el grupo de los "fops" (Espinoza et al., 2002). En un biotipo de L. multiflorum se determinó la presencia de resistencia a glifosato, a inhibidores de la acetolactato sintasa (ALS) y de la ACCasa incluyendo el cletodim (Diez de Ulzurrun y Leaden, 2012).

Actualmente se encuentran reportados diversos biotipos de $P$. аппиа con resistencia a herbicidas triazínicos, ureas sustituidas (estos dos grupos afectan 
la fotosíntesis en el fotosistema II), dinitroanilinas, glifosato y paraquat principalmente (Eelen et al., 1999; Kelly et al., 1999; Owen, 2001; Isgrigg et al., 2002).

Como se mencionó anteriormente, $P$. апnиa se encuentra entre las malezas más problemáticas y comunes para la siembra de cebolla en la zona de Cartago (Araya, 2000). Para su control se ha utilizado cletodim, herbicida recomendado para el control de poáceas en posemergencia y con uso autorizado en cebolla (SFE, 2014), ya que es selectivo a este cultivo. Inicialmente este herbicida se empleó con buen éxito, pero paulatinamente se han presentado fallas o deficiencias en el control de esta maleza en algunas fincas que usan, la dosis comercial recomendada, lo que hace suponer (por este comportamiento), que existen ya biotipos de $P$. апnиa con resistencia a este herbicida. Debido a lo anterior, se planteó como objetivo de este trabajo verificar la presencia de resistencia al cletodim en poblaciones de $P$. апnиa, en el cultivo de cebolla en la zona alta de Cartago, Costa Rica.

\section{MATERIALES Y MÉTODOS}

\section{Evaluación de resistencia al cletodim de dos poblaciones de Poa annua provenientes de dos fincas de Tierra Blanca, Cartago. 2007}

\section{Ubicación}

El trabajo se realizó en el invernadero de las instalaciones de la Estación Experimental Fabio Baudrit Moreno (EEFBM), situada en la provincia de Alajuela a $840 \mathrm{msnm}$, coordenadas latitud $\mathrm{N}$ $10^{\circ} 00.373^{\prime}$ y longitud W $084^{\circ} 15.992^{\prime}$.

\section{Procedimiento}

En octubre del 2006 en Tierra Blanca de Cartago se colectaron plantas de $P$. апnиa provenientes de dos fincas, una donde se presentaban problemas de control de esta maleza con cletodim (R) y de otra finca vecina donde el control era satisfactorio (S). Estas se trasladaron a la Estación Experimental Fabio Baudrit Moreno (EEFBM) y se trasplantaron a recipientes plásticos con el fin de obtener semilla para las pruebas posteriores de resistencia al cletodim, en condiciones de invernadero. A inicios del 2007, se realizó la prueba de resistencia, para lo cual se sembraron las semillas obtenidas en un almácigo; se trasplantaron seis plántulas de $P$. апnиa de tamaño uniforme por recipiente plástico con capacidad de cuatro litros y se les dio un período de establecimiento de dos semanas. Posteriormente, se les aplicaron los tratamientos con el herbicida. Los recipientes se mantuvieron dentro de una casa de mallas y únicamente se les aplicó riego cada dos días para mantener el suelo cercano a la capacidad de campo. No se aplicaron fertilizantes ni otros productos químicos.

\section{Aplicación de los tratamientos}

A las plántulas de $P$. апnиa provenientes de las dos fincas se aplicaron dosis crecientes del herbicida cletodim cuando las mismas tenían de tres a cinco hojas, edad recomendada para una mayor efectividad del herbicida (Picado y Ramírez, 1998). El herbicida se aplicó con un equipo aspersor AZ accionado con $\mathrm{CO}_{2}$ a una presión constante de 2 bares y calibrado para aplicar 200 1/ha. Una vez aplicado el herbicida, se hizo una evaluación preliminar a los veinte días después de la aplicación (dda) y la definitiva a los 30 dda.

\section{Diseño experimental}

El experimento consistió en la aplicación de cuatro dosis del herbicida y un testigo, para cada una de las poblaciones de P. апnиа (Cuadro 1). Se utilizó un diseño irrestricto al azar que constó de diez tratamientos y cinco repeticiones. Cada recipiente constituyó la unidad experimental. En cada evaluación se contó el número de plántulas muertas o con la hoja bandera muerta (hoja más alta por debajo de la panícula) (U.P., 1975); además se calculó el porcentaje de sobrevivencia. Para el análisis de los datos se empleó un ANDEVA con análisis factorial y las medias se separaron por medio de la prueba DMS al 5\%. Los análisis estadísticos fueron realizados con el software libre "Anawin 2012". 
Cuadro 1. Descripción de los tratamientos utilizados para evaluar la presencia de poblaciones resistentes de Poa annua. Alajuela (2007) y Cartago (2009), Costa Rica.

Table 1. Description of treatments applied to assess the presence of resistant Poa аппиа populations. Alajuela (2007) and Cartago (2009).

\begin{tabular}{|c|c|c|c|c|c|}
\hline \multicolumn{3}{|c|}{ Experimento 1, Alajuela } & \multicolumn{3}{|c|}{ Experimento 2, Cartago } \\
\hline Tratamiento & $\begin{array}{c}\text { Biotipo de } \\
\text { P. annua }(\text { Ro S) }\end{array}$ & $\begin{array}{l}\text { Dosis de cletodim* } \\
\quad(\mathrm{g} \text { i.a./ha)*** }\end{array}$ & Tratamiento & $\begin{array}{c}\text { Biotipo de } \\
\text { P. annua }(\operatorname{Ro} S) \\
\end{array}$ & $\begin{array}{l}\text { Dosis de cletodim* } \\
\text { (g i.a./ha) }\end{array}$ \\
\hline & $\begin{array}{l}\text { Población conside- } \\
\text { rada resistente }(\mathrm{R})\end{array}$ & & & $\begin{array}{l}\text { Población conside- } \\
\text { rada resistente }(\mathrm{R})\end{array}$ & \\
\hline $\mathrm{T} 1$ & Testigo R & 0 & $\mathrm{~T} 1$ & Testigo R & 0 \\
\hline $\mathrm{T} 2$ & Población R & 60 & $\mathrm{~T} 2$ & Población R & 120 \\
\hline $\mathrm{T} 3$ & Población R & 120 & $\mathrm{~T} 3$ & Población R & 240 \\
\hline $\mathrm{T} 4$ & Población R & 180 & $\mathrm{~T} 4$ & Población R & 480 \\
\hline \multirow[t]{2}{*}{$\mathrm{T} 5$} & Población R & 240 & $\mathrm{~T} 5$ & Población R & 960 \\
\hline & & & T6 & Población R & 1920 \\
\hline & $\begin{array}{l}\text { Población conside- } \\
\text { rada susceptible (S) }\end{array}$ & & & $\begin{array}{l}\text { Población conside- } \\
\text { rada susceptible (S) }\end{array}$ & \\
\hline $\mathrm{T} 6$ & Testigo $\mathrm{S}$ & 0 & $\mathrm{~T} 7$ & Testigo $\mathrm{S}$ & 0 \\
\hline $\mathrm{T} 7$ & Población S & 60 & $\mathrm{~T} 8$ & Población S & 120 \\
\hline $\mathrm{T} 8$ & Población S & 120 & T9 & Población S & 240 \\
\hline T9 & Población S & 180 & $\mathrm{~T} 10$ & Población S & 480 \\
\hline \multirow[t]{2}{*}{$\mathrm{T} 10$} & Población S & 240 & $\mathrm{~T} 11$ & Población S & 960 \\
\hline & & & $\mathrm{T} 12$ & Población S & 1920 \\
\hline
\end{tabular}

* El producto formulado utilizado en estos experimentos fue Select 12 EC (la indicación del nombre comercial no indica ninguna preferencia o recomendación) / The formulated product used in these experiments was Select 12 EC (the instruction of the trade name does not show any preference or recommendation).

** Se refiere a la cantidad de gramos de ingrediente activo aplicados por hectárea / It referes to the amount (in grams) of the active ingredient applied per hectare.

\section{Evaluación de resistencia al cletodim de dos poblaciones de $P$. annua provenientes de una misma finca en Prusia, Cartago. 2009}

\section{Ubicación}

El trabajo se realizó en las instalaciones del Ministerio de Agricultura y Ganadería (MAG), situadas en Potrero Cerrado de Cartago a 2072 msnm.

\section{Procedimiento}

Se llenaron sesenta recipientes plásticos con capacidad de un litro con suelo franco (con concentraciones de arena, limo y arcilla de 50,33 y $17 \%$ respectivamente) procedente de una finca situada en la zona de Prusia, a $2342 \mathrm{msnm}$, con coordenadas latitud N $09^{\circ} 55.833^{\prime \prime}$ y longitud O $083^{\circ} 53.191^{\prime}$. Los recipientes se llenaron con suelo hasta aproximadamente $2 \mathrm{~cm}$ por debajo del borde. Luego, la mitad de estos se sembraron con semilla de $P$. апnиа proveniente de un área donde el agricultor no tenía problemas para controlar esta especie con cletodim (población S) y la otra mitad con semilla de otra área donde existían problemas para su control (población R). Posteriormente las semillas se taparon con suelo hasta el borde del recipiente.

\section{Aplicación de los tratamientos}

A las plantas de $P$. апnиa se les aplicó el herbicida cletodim cuando tenían de dos a tres hojas y una altura 
entre uno a tres $\mathrm{cm}$. La aplicación se realizó con un aspersor de espalda de dieciséis litros, utilizando una boquilla 8002 con regulador de presión a 2 bares, el volumen de aplicación fue de $250 \mathrm{1} / \mathrm{ha}$. El uso del regulador permitió mantener una presión constante y por lo tanto una aplicación uniforme. Los recipientes se mantuvieron expuestos al ambiente natural y recibieron riegos cuando fue necesario para mantener las plantas en adecuadas condiciones de crecimiento.

\section{Diseño experimental}

Se utilizó un diseño experimental de bloques completos al azar con doce tratamientos y cinco repeticiones (un recipiente por repetición) (Cuadro 1). Las variables evaluadas fueron el porcentaje de control de $P$. апnиa y el porcentaje de plantas vivas (macollas) a los 15 y 30 días después de la aplicación (dda). El porcentaje de control se evaluó comparando el control logrado en cada tratamiento en relación con el testigo sin aplicar, y el porcentaje de sobrevivencia de $P$. апnиа, con relación a la población inicial. Se realizó posteriormente un ANDEVA con análisis factorial, las medias se separaron por medio de la prueba de DMS al 5\%. Los datos fueron analizados con el "software" libre "Anawin 2012".

Para determinar si un biotipo o una población de una especie es resistente se utiliza el GR $_{50}$ (siglas en inglés), y corresponde a la dosis del ingrediente activo del producto que es capaz de disminuir en un $50 \%$ la biomasa del biotipo en estudio (Duarte, 2015). Sin embargo, en este trabajo se utilizó el porcentaje de sobrevivencia de los biotipos en estudio, como indicador de resistencia. Para poder comparar el grado de resistencia entre biotipos o poblaciones de la misma especie se utiliza el $\mathrm{IR}_{50}$ : índice generado por la división del $\mathrm{GR}_{50}$ de la población resistente entre el $\mathrm{GR}_{50}$ de la población susceptible para cada herbicida que se quiera evaluar (Jalaludin y Powles, 2014).

\section{RESULTADOS Y DISCUSIÓN}

\section{Evaluación de resistencia a cletodim de dos poblaciones de $P$. annua provenientes de dos fincas en Tierra Blanca, Cartago. 2007}

En el primer experimento hubo diferencias significativas entre ambas poblaciones de $P$. аппиа.
Las plantas de la población considerada susceptible (S) fueron eliminadas completamente con la dosis comercial (120 g i.a.); mientras que en la población considerada con posible resistencia (R), sobrevivió más del $40 \%$ de las plantas a la dosis de 120 y 180 g i.a. (Figura 1 ).

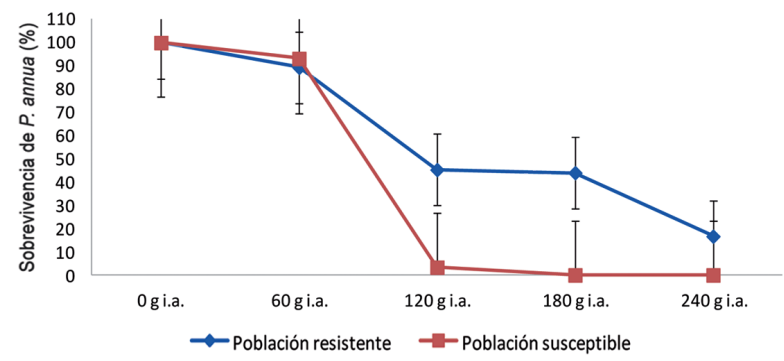

Figura 1. Porcentaje de sobrevivencia de dos poblaciones de Pоа аппиа a diferentes dosis del herbicida cletodim. Estación Experimental Fabio Baudrit Moreno (EEFBM), Alajuela, Costa Rica. 2007.

Figure 1. Survival percentage of two Pоа аппиа populations at different clethodim doses. Fabio Baudrit Moreno Agricultural Experiment Station (EEAFBM), Alajuela, Costa Rica. 2007.

En la población susceptible se observó que el cletodim a la mitad de la dosis comercial no tuvo efecto negativo sobre las plantas de P. апnиа, mientras que a la dosis comercial recomendada no hubo sobrevivencia de plantas (Figura 1). Esto indica que la dosis recomendada para el herbicida es la adecuada para combatir las poblaciones susceptibles. A la vez, los resultados obtenidos indican que una sub-dosificación podría provocar la sobrevivencia de las plantas, por un control deficiente de las mismas.

Con respecto a la población $\mathrm{R}$, incluso al doble de la dosis comercial (2X) hubo un $17 \%$ de plantas sobrevivientes (Figura 1). Se observó en forma clara que al utilizar dosis del herbicida de manera creciente en la población $\mathrm{R}$, tienden a bajar los porcentajes de sobrevivencia en una forma casi proporcional (Figura 2), aún así a la dosis de $2 \mathrm{X}$ el porcentaje de plantas sobrevivientes, se puede considerar importante, teniendo en cuenta la cantidad de semillas que produce P. аппиа (Lush, 1988a; 1988b). Estos resultados explican en parte, porque en algunas áreas productoras de cebolla el control de P. апnиа con dosis comerciales de cletodim resulta deficiente. A la vez, en estas condiciones no se debería continuar con el uso de este 

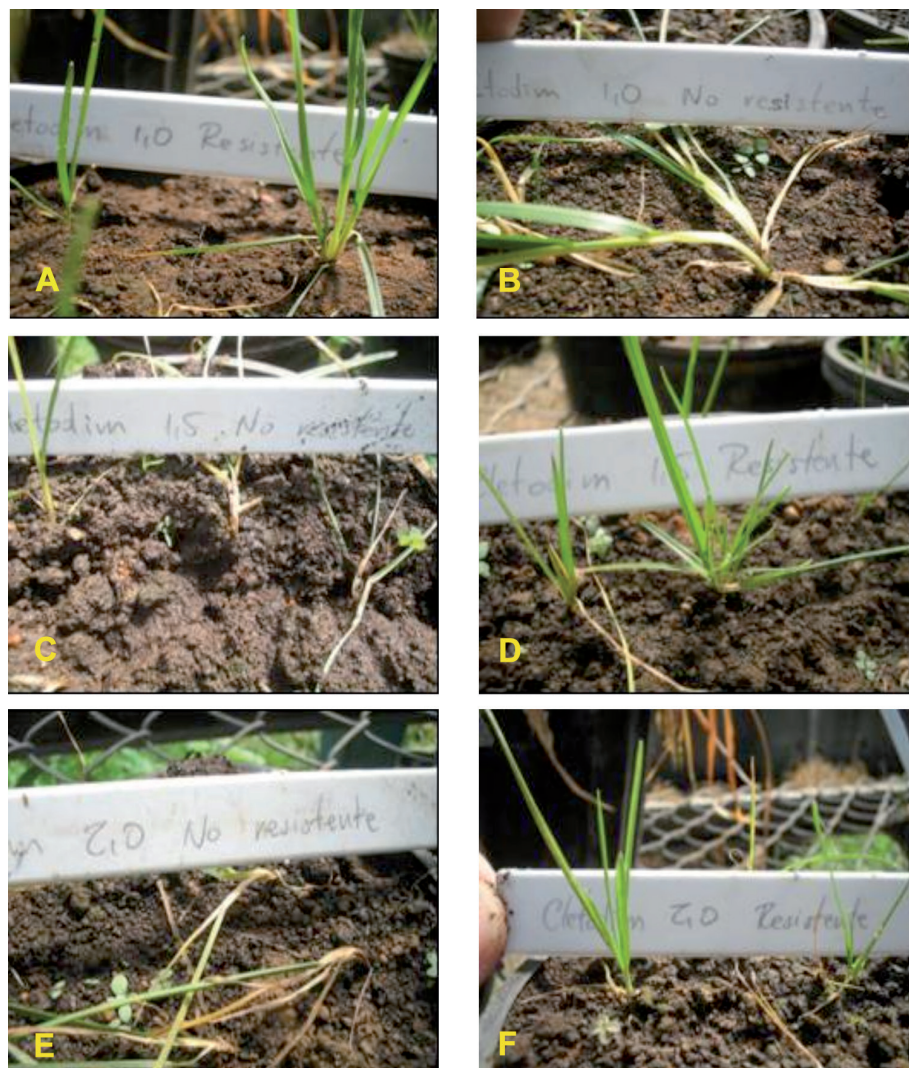

Figura 2. Plántulas de $P$. аппиа al momento de la evaluación final por su resistencia al herbicida cletodim. A. 1,0x resistente. B. 1,0x susceptible. C. 1,5x susceptible. D. 1,5x resistente. E. 2,0x susceptible. F. 2,0x resistente. EEFBM, Alajuela, Costa Rica. 2007.

Figure 2. $P$. аппиа seedlings at the moment of the final evaluation of their resistance to clethodim. A. 1.0x resistant. B. 1.0x susceptible. C. 1.5x susceptible. D. 1.5x resistant. E. 2.0x susceptible, F. 2.0x resistant. EEAFBM, Alajuela, Costa Rica. 2007.

herbicida para no aumentar la presión de selección sobre esas plantas que muestran resistencia.

La presencia de $P$. апnиa en zonas de cultivos hortícolas del país representa un problema serio para los productores, en especial en cebolla, por la poca competencia que ofrece este cultivo con relación a las malezas. Encontrar plantas de P. аппиа con resistencia al cletodim, producto recomendado para su control y permitido para usar en el cultivo de cebolla, indica la necesidad de nuevas evaluaciones de herbicidas para el manejo de esta maleza en dicho cultivo, máxime la alta variabilidad genética y la alta cantidad de semillas que produce esta especie (Lush, 1988a; 1988b; Eelen et al., 1999).
Evaluación de resistencia al cletodim de dos poblaciones de Poa annua provenientes de una misma finca en Prusia, Cartago. 2009

\section{Porcentaje de control de Poa апnиa}

La población $\mathrm{S}$ fue susceptible al cletodim en las dosis evaluadas, con altos porcentajes de control a partir de la dosis comercial utilizada, resultados que coindicen con lo observado en el experimento previo para esta población. Por otro lado, en la población $\mathrm{R}$ se registró un control pobre aún con la dosis donde se duplicó la cantidad más alta de ingrediente activo utilizada en el primer experimento (cuatro veces 
la dosis normal de control). El efecto de la dosis/ dependencia para alcanzar un buen control es claro. Si bien, se observó un incremento en el porcentaje de control con las dosis más elevadas, ese efecto es insuficiente desde el punto de vista técnico y resultaría además muy costoso. Las plantas de esta población tratadas con dosis menores se recuperaron al cabo de treinta días después de la aplicación, por lo que el porcentaje de control fue muy bajo. Solo con la dosis de $1920 \mathrm{~g}$ i.a./ha se logró mantener un porcentaje de control aceptable (73\%) a los 30 dda (Cuadro 2).

En pruebas de laboratorio, a nivel de germinación, el biotipo de $P$. аппиа "Melle" resistente a triazínicos fue menos sensible a haloxifop y cletodim. Sin embargo, esta respuesta no fue confirmada en aplicaciones de posemergencia con estos herbicidas (Eelen et al., 1999).

$\mathrm{Se}$ encontraron interacciones entre las dos poblaciones y las distintas dosis evaluadas (principalmente a los 15 dda). Sin embargo, en ambas evaluaciones el porcentaje de control fue mayor en la población $\mathrm{S}$ (en todas las dosis), mientras que en la población R solo se logró un control regular con la dosis más alta. Para que una especie se considere maleza resistente, debe presentarse en cantidades suficientes para afectar el cultivo y que los biotipos que exhiben niveles bajos de resistencia ofrezcan problemas de control en el campo (Taberner et al., 2007; Valverde y Heap, 2010); situación que empieza a ocurrir con $P$. апnиa en las plantaciones de cebolla de la zona alta de
Cartago. Estos resultados indican que en la población $\mathrm{R}$ analizada hay presencia de individuos con resistencia al cletodim, ya que a la dosis $1 \mathrm{X}$ el porcentaje de control fue de 22 y $2 \%$ a los 15 y a los 30 dda respectivamente. Además, para obtener el 50\% de control del biotipo resistente se requiere entre ocho a dieciséis veces de la dosis normal recomendada.

\section{Porcentaje de sobrevivencia de plantas de Роа аппиа}

Todos los tratamientos partieron de poblaciones estadísticamente similares de $P$. аппиа (datos no incluidos). Al evaluar el porcentaje de sobrevivencia a los 15 dda (Cuadro 3) se observaron diferencias para cada una de las dos poblaciones ( $\mathrm{S}$ y R) y entre estas. En el caso de la población susceptible la sobrevivencia fue menor al compararse con el testigo y conforme aumentó la dosis del producto; en el caso de la población $\mathrm{R}$ se presentó disminución de sobrevivencia en el testigo y también dentro de los tratamientos aplicados, conforme se aumentó la dosis del producto.

A los 30 dda el comportamiento fue similar al que se observó en la primera evaluación, ya que se llegó a una sobrevivencia de cero cuando se utilizó la dosis de $3 \mathrm{X}$ en la población $\mathrm{S}$, en tanto que en la población $\mathrm{R}$ la sobrevivencia fue mayor que en la población $\mathrm{S}$, con tendencia a disminuir conforme la dosis creció. Otra observación en estos resultados es la disminución

Cuadro 2. Porcentaje de control de dos biotipos de Poa апnиа, resistente (R) y susceptible (S), luego de la aplicación de cinco dosis de cletodim. Cartago, Costa Rica. 2009.

Table 2. Percentage control of two Poa аппиа biotypes, resistant (R) and susceptible (S), after spraying five doses of clethodim. Cartago, Costa Rica. 2009.

\begin{tabular}{ccccc}
\hline \multirow{2}{*}{$\begin{array}{c}\text { Dosis de cletodim } \\
(\mathbf{g} / \mathbf{h a})\end{array}$} & \multicolumn{2}{c}{$\mathbf{1 5}$ dda } & \multicolumn{2}{c}{ 30 dda } \\
\cline { 2 - 5 } & Biotipo $\mathbf{S}$ & Biotipo $\mathbf{R}$ & Biotipo $\mathbf{~}$ & Biotipo R \\
\hline 120 & $93 \mathrm{Aa} *$ & $22 \mathrm{Bbc}$ & $83 \mathrm{Aa}$ & $2 \mathrm{Bc}$ \\
240 & $92 \mathrm{Aa}$ & $15 \mathrm{Bc}$ & $93 \mathrm{Aa}$ & $3 \mathrm{Bc}$ \\
480 & $98 \mathrm{Aa}$ & $30 \mathrm{Bb}$ & $100 \mathrm{Aa}$ & $7 \mathrm{Bc}$ \\
960 & $99 \mathrm{Aa}$ & $83 \mathrm{Ba}$ & $100 \mathrm{Aa}$ & $42 \mathrm{Bb}$ \\
1920 & $99 \mathrm{Aa}$ & $76 \mathrm{Ba}$ & $100 \mathrm{Aa}$ & $73 \mathrm{Ba}$ \\
\hline
\end{tabular}

*Medias con igual letra mayúscula en la misma fila o igual letra minúscula en la misma columna no difieren entre sí según la prueba de DMS $(\mathrm{P}<0,05 \%)$ / Means with the same capital letter in the same row or same lower- case letter in the same column do not show any differences (DMS test, $\mathrm{P}<0,05 \%)$.

dda: días después de la aplicación / dda: days after application. 
Cuadro 3. Efecto de la aplicación a diferentes dosis del herbicida cletodim sobre el porcentaje de sobrevivencia de dos poblaciones: resistente (R) y susceptible (S) de Poa annua. Cartago, Costa Rica. 2009.

Table 3. Effect of the application of clethodim doses on the survival percentage of two Poa аппиа populations, resistant (R) and susceptible (S). Cartago, Costa Rica. 2009.

\begin{tabular}{lcccc}
\hline \multirow{2}{*}{$\begin{array}{l}\text { Dosis de cletodim } \\
\text { g/ha) }\end{array}$} & \multicolumn{2}{c}{$\mathbf{1 5}$ dda } & \multicolumn{2}{c}{30 dda } \\
\cline { 2 - 5 } & Población $\mathbf{S}^{*}$ & Población $\mathbf{R}^{*}$ & Población $\mathbf{S}^{*}$ & Población R* \\
\hline Testigo & $77,57 \mathrm{Aa}$ & $59,42 \mathrm{Bbc}$ & $79,91 \mathrm{Aa}$ & $56,16 \mathrm{Bab}$ \\
120 & $37,54 \mathrm{Bb}$ & $82,88 \mathrm{Aa}$ & $19,8 \mathrm{Bb}$ & $63,48 \mathrm{Aa}$ \\
240 & $37,1 \mathrm{Bb}$ & $68,59 \mathrm{Aab}$ & $6,86 \mathrm{Bbc}$ & $52,27 \mathrm{Aab}$ \\
480 & $10,09 \mathrm{Bc}$ & $61,08 \mathrm{Abc}$ & $0 \mathrm{Bc}$ & $47,14 \mathrm{Ab}$ \\
960 & $6,19 \mathrm{Bc}$ & $48,84 \mathrm{Acd}$ & $2,00 \mathrm{Bc}$ & $40,83 \mathrm{Ab}$ \\
1920 & $8,48 \mathrm{Bc}$ & $39,13 \mathrm{Ad}$ & $3,64 \mathrm{Ac}$ & $12,66 \mathrm{Ac}$ \\
\hline
\end{tabular}

* Medias con igual letra mayúscula en la misma fila o igual letra minúscula en la misma columna no difieren entre sí según la prueba de DMS $(\mathrm{P}<0,05 \%)$ / Means with the same capital letter in the same row or same lower- case letter in the same column do not show any differences (DMS test, $\mathrm{P}<0,05 \%)$.

dda: días después de la aplicación / dda: days after the application.

de sobrevivencia de los tratamientos testigos para las dos poblaciones, este hecho se debe posiblemente a la competencia intraespecífica que se presenta dentro de las poblaciones de las especies y que tiende a disminuir conforme alcanzan su equilibrio; este proceso podría haber afectado a los tratamientos aplicados pero en menor grado de lo que lo hicieron las aplicaciones del producto (Cuadro 3). En el caso de los testigos, se tiene que la población tiende a mantenerse de una evaluación a otra. Para el testigo susceptible se tiene un porcentaje un poco mayor al final, posiblemente por la germinación escalonada de algunas semillas, característica propia de las malezas.

La sobrevivencia de algunas plantas de $P$. апnиa en la población $S$, aun a dosis altas, hace suponer que aunque fue susceptible, ya hay presencia de plantas con resistencia. De igual manera en la población $\mathrm{R}$, también se presentaron algunos individuos susceptibles. En el caso de la población $\mathrm{S}$, al encontrarse en la misma finca que la población $\mathrm{R}$, puede darse el transporte involuntario de semillas de plantas resistentes al prepararse el suelo, en las herramientas de trabajo o por los mismos trabajadores. En el caso de la población $\mathrm{R}$, al tratarse de una especie con una alta variabilidad genotípica y fenotípica (Holm et al., 1997; Eelen et al., 1999; Isgrigg et al., 2011), es posible que se presenten individuos susceptibles y casos de segregación.
También, si se compara la sobrevivencia de individuos de $P$. аппиа tratados con la misma dosis, entre el experimento 1 y el 2 , se puede inferir que hubo mayor sobrevivencia en el segundo experimento realizado dos años después, donde se requirió $16 \mathrm{X}$ de la dosis para llegar a un $12 \%$ de plantas que sobrevivieron; comportamiento esperable si se considera que los productores de cebolla siguieron en ese periodo utilizando el cletodim, lo que pudo favorecer la presión de selección hacia individuos con resistencia.

Estos resultados concuerdan con lo observado en la evaluación del porcentaje de control (Cuadro 2), además, es congruente con lo observado en campo por diferentes agricultores, quienes manifiestan la dificultad de controlar algunas poblaciones de $P$. аппиа aún aumentando las dosis del cletodim.

Determinación del índice de resistencia del biotipo de Poa annua resistente

En este estudio la estimación del $\mathrm{GR}_{50}$ para el cletodim, en las dos poblaciones se hizo utilizando los valores de porcentaje de sobrevivencia. En el caso de la población $\mathrm{R}$ se encontró que la dosis que redujo en un $50 \%$ la sobrevivencia fue de 1338,90 g i.a./ha (Cuadro 4). En tanto que en la población $\mathrm{S}$, el $\mathrm{GR}_{50}$ estimado fue de 54,15 g i.a./ha, menos de la mitad de la dosis 
Cuadro 4. Estimación del $\mathrm{GR}_{50}$ y el índice de resistencia $\left(\mathrm{GR}_{50} \mathrm{R} / \mathrm{GR}_{50} \mathrm{~S}\right)$ para el cletodim, en dos poblaciones de Poa annua: resistente (R) y susceptible (S). Cartago, Costa Rica. 2009.

Table 4. Estimate of $\mathrm{GR}_{50}$ and resistant index $\left(\mathrm{GR}_{50} \mathrm{R} / \mathrm{GR}_{50} \mathrm{~S}\right)$ for clethodim over two Poa annua populations, resistant (R) and susceptible (S). Cartago, Costa Rica. 2009.

\begin{tabular}{lllccc}
\hline Población & Herbicida & Regresión & R $^{\mathbf{2}}$ & GR $_{\mathbf{5 0}}$ & IR \\
\hline Resistente & Cletodim & $28,08=60,3474+(-0,0241) X$ & 0,9515 & 1338,896 & 24,728 \\
Susceptible & Cletodim & $39,95=75,2948+0,0896$ X $+(-5,4627) X^{\wedge} 0,5$ & 0,9557 & 54,145 & \\
\hline
\end{tabular}

IR (índice de resistencia) $=\mathrm{GR}_{50}$ Población R/GR ${ }_{50}$ Población S / IR (resistant index) $=\mathrm{GR}_{50}$ Population R/GR ${ }_{50}$ Population S.

comercial recomendada que es de $120 \mathrm{~g}$ i.a./ha. El índice de resistencia (IR) estimado para la población de $P$. annua considerada resistente fue de 24,7; este dato indica que dicha población presentó 24,7 veces más resistencia que la población susceptible.

La existencia de resistencia en diferentes biotipos de $P$. annua a varios grupos de herbicidas con distintos modos de acción, ya fue demostrada, lo mismo que la resistencia a herbicidas del grupo de las ACCasa por parte de diferentes poáceas (Espinoza et al., 2002; Volenberg y Stoltenberg, 2002; Diez de Ulzurrun y Leaden, 2012). Se podría estar ante un nuevo biotipo de $P$. annua con presencia de resistencia a inhibidores de la ACCasa. Volenberg y Stoltenbert (2002), consideran por ejemplo, que la resistencia encontrada en accesiones de $S$. faberi y D. sanguinalis se debe a una alteración de la enzima ACCasa.

\section{LITERATURA CITADA}

Araya, E.E. 2000. Control químico de Polygonum aviculare y otras malezas en el cultivo de cebolla (Allium cepa L.). Tesis Lic. Ing. Agr., Universidad de Costa Rica, Facultad de Agronomía, San José, CRC.

Binkholder, K.M., B.S. Fresenburg, T.C. Teuton, X. Xiong, and R.J. Smeda. 2011. Selection of glyphosateresistant annual bluegrass (Poa апnиа) on a golf course. Weed Sci. 59:286-289.

Diez de Ulzurrun, P., y M.I. Leaden. 2012. Análisis de la sensibilidad de biotipos de Lolium multiflorum a herbicidas inhibidores de la enzima ALS, ACCasa y glifosato. Planta Daninha 30:667-673.

Duarte, N. 2015. Resistencia del biotipo RCCR 1 de Rottboellia cochinchinensis (Lour.) W.D. Clayton. a herbicidas inhibidores de la acetil coenzima A
- carboxilasa (ACCasa). Tesis Ing. Agr., Facultad de Ciencias Agroalimentarias, Universidad de Costa Rica, San José, CRC.

Eelen, H., R. Bulcke, B. Debussche, and I. Heap. 1999. Annual bluegrass (Poа annua L.): a challenging weed. $12^{\text {th }}$ Australian Weeds Conference. 12 - 16 september 1999. AUS. p. 62-66.

Espinoza, N., K. Seitz, M. Mera., C. Jobet, J. Díaz, y R. De Prado. 2002. Respuesta a herbicidas ACCasa y ALS de un biotipo de Lolium rigidum. http://www.sach.cl/revista/ pdf/SIMIENTE_72_3_4.pdf (consultado 16 ene. 2013).

Heap, I. 2014. International survey of herbicide resistant weeds. http://www.weedscience.org (accessed 12 may 2014).

Holm, L.R.G., D.L. Pluckenet, J.V. Pancho, and J.P. Herberger. 1977. The world's worts weeds. Distribution and biology. Krieger Publishing Company, Malabar, FL, USA.

Isgrigg, J., F.H. Yelverron, C. Brownie, and I.S. Warren. 2002. Dinitroaniline resistant annual bluegrass (Poa апnиа). Weed Sci. 50:86-90.

Jalaludin, Q., and S.B. Powles. 2014. Multiple resistance across glufosinate, glyphosate, paraquat and ACCaseinhibiting herbicides in an Eleusine indica population. Weed Research. http://dx.doi.org/10.1111/wre.12118 (accessed 21 apr. 2015).

Kelly, S.T., G.E. Coats, and D.S. Luthe. 1999. Mode of resistance of triazine-resistant annual bluegrass (Poa annua). Weed Technol. 13:747-752.

Lush, W.M. 1988a. Biology of Poa annua in a temperate zone golf putting green (Agrostis stolonifera/Poa апnua). I. The above-ground population. J. Appl. Ecol. 25:977-988.

Lush, W.M. 1988b. Biology of Poa annua in a temperate zone golf putting green (Agrostis stolonifera/Poa апnиа). II. The seed bank. J. Appl. Ecol. 25:989-997. 
Owen, M.D. 2001. World maize/soybean. And herbicide resistance. In: S.B. Powles, and D.L. Shaner, editors, Herbicide resistance and world grains. CRC Press, NJ, USA. http://www.crenetbase.com/doi/ pdfplus/10.1201/9781420039085.ch4 (accessed 31 oct. 2013).

Picado, J., y F. Ramírez. 1998. Guía de agroquímicos. EDISA, San José, CRC.

Pohl, R.W. 1980. Flora costarricensis, Family \# 15, Gramineae. Field Museum of Natural History, Illinois, USA.

Preston, C., and C.A. Mallory-Smith. 2001. Biochemical mechanisms, inheritance, and molecular genetics of herbicide resistance in weeds. In: S.B. Powles, and D.L. Shaner, editors, Herbicide resistance and world grains. CRC Press, NJ, USA. http://www.crenetbase. com/doi/pdf/10.1201/9781420039085.ch2 (accessed 14 jan. 2013).

Ryan, G.F. 1970. Resistance of common groundsel to simazine and atrazine. Weed Sci. 18:614-616.

SFE (Sistema Fitosanitario del Estado). 2014. Consulta sobre productos registrados. https://www.sfe.go.cr/ insumosys/Principal.htm (consultado 1 jul. 2014).
Taberner, A., A. Cirujeda, y C. Zaragoza, C. 2007. Manejo de malezas resistentes a herbicidas. 100 preguntas sobre resistencias. FAO (Organización de las Naciones Unidas para la Agricultura y la Alimentación, IT). ftp://ftp.fao.org/docrep/fao/010/a1422s/a1422s00.pdf (consultado 5 feb. 2013).

UP (University of the Philippines). 1975. Cultivo del arroz, manual de producción. Agustín Contin (trad.). Limusa, México, D.F., MEX.

Valverde, B., e I.M. Heap. 2010. El estado actual de la resistencia a herbicidas en el mundo. http://www. inia.cl/medios/biblioteca/serieactas/NR36351.pdf (consultado 12 nov. 2012).

Volenberg, D., and D. Stoltenberg. 2002. Altered acetylcoenzyme carboxylase confers resistance to clethodim, fluazifop and sethoxidim in Setaria faberi and Digitaria sanguinalis. Weed Res. 42:342-350.

WSSA (Weed Science Society of America). 1998. "Herbicide resistance" and "herbicide tolerance" defined. Weed Technol. 12:789. 